Classification

Physics Abstracts

$23.70 \mathrm{j}$

\title{
Dynamique de la désintégration pour trois types de processus nucléaires
}

\author{
T. Benfoughal $\left({ }^{1}\right)$ et M. Mirea $\left({ }^{2}\right)$ \\ ( ${ }^{1}$ Institut de Physique Nucléaire, 91406 Orsay Cedex, France \\ $\left({ }^{2}\right)$ Institut de Physique Atomique, Bucarest, Roumanie
}

(Reçu le 20 janvier 1995, accepté le 4 avril 1995)

\begin{abstract}
Résumé. - Afin d'étudier le comportement dynamique de trois modes de désintégration nucléaire (fission froide, désintégration alpha et radioactivité par émission d'ions lourd), nous utilisons une méthode numérique basée sur les équations d'Euler-Lagrange pour obtenir la trajectoire dynamique optimale dans un espace de configuration à trois dimensions. Les degrés de liberté du système retenus sont l'élongation, la taille du col et l'assymétrie de masse. Pour le calcul de l'intégrale d'action par la méthode WKB, l'inertie du système est déterminée dans l'approximation Werner-Wheeler, et l'énergie de déformation est calculée dans le cadre du modèle de la goutte liquide pour une interaction nucléaire de type Yukawa-plus-exponentielle, étendu aux systèmes binaires avec des densités de charge différentes. Cette méthode est appliquée à l'étude de trois modes de désintégration du $\mathrm{Pu}-238$ : désintégration alpha, radioactivité par émission du Si-32 et fission froide avec, pour fragment léger, le Mo-104.
\end{abstract}

\begin{abstract}
In order to study the dynamic behaviours of three disintegration modes (cold fission, alpha-decay and cluster radioactivities), we use a numerical method based on the EulerLagrange's equation of motion to obtain a constrained minimum of the WKB-integral in a tridimensional configuration space. The inertia is determined using the Werner-Wheeler approximation and the energy is computed in agreement with the Yukawa-plus-exponential model extended for binary systems with different charge densities. The degrees of freedom of the system are: elongation, necking-in and mass-asymmetry. Least-action trajectories are found for three decay-modes of ${ }^{238} \mathrm{Pu}$.
\end{abstract}

\section{Introduction}

L'intérêt de développer les modèles de fission dans un domaine très large d'assymétries de masse est principalement dû à l'étude de l'émission de noyaux exotiques. Ce nouveau mode de désintégration nucléaire spontanée, caracterisé par une très grande assymétrie de masse, a été prédit $[1,2]$ en 1980 , au moyen de trois modèles phénoménologiques simples basés sur un traitement unifié de la désintégration alpha, de l'émission de noyaux exotiques et de la fission froide. Quelques années plus tard, en 1984, l'expérience de Rose et Jones [3] a mis en évidence la 
désintégration $d u^{223} \mathrm{Ra}$ avec, pour fragment léger émis, le ${ }^{14} \mathrm{C}$. Récemment encore, on a observé une vague de découvertes expérimentales concernant ce nouveau type d'émission. A présent, la communauté scientifique dispose d'informations expérimentales [4] pour les émissions d'ions lourds suivants : ${ }^{14} \mathrm{C}$ produit dans la désintégration des noyaux ${ }^{221} \mathrm{Fr},{ }^{221-224,226} \mathrm{Ra}$ et ${ }^{225} \mathrm{Ac}$; ${ }^{20} \mathrm{O}$ dans celle du noyau ${ }^{228} \mathrm{Th} ;{ }^{23} \mathrm{~F}$ dans celle du noyau ${ }^{231} \mathrm{~Pa} ;{ }^{24} \mathrm{Ne}$ dans celle des noyaux ${ }^{230} \mathrm{Th},{ }^{231} \mathrm{~Pa}$ et ${ }^{232-234} \mathrm{U} ;{ }^{28} \mathrm{Mg}$ dans celle des noyaux ${ }^{234} \mathrm{U}$ et ${ }^{236,238} \mathrm{Pu} ;{ }^{32} \mathrm{Si}$ dans celle du noyau ${ }^{238} \mathrm{Pu}$ et ${ }^{34} \mathrm{Si}$ dans celle du noyau ${ }^{242} \mathrm{Cm}$.

A Orsay, une structure fine dans la distribution de l'énergie cinétique observée dans l'émission $d u{ }^{14} \mathrm{C}$ a été décelée [5] ; ce qui met en évidence le rôle joué par les effets microscopiques durant le processus.

A côté des calculs macroscopiques qui ont conduit aux prédictions de ce nouveau type de radioactivité, les théories microscopiques [6] ont décrit, quelquefois avec succès, ces désintégrations par une généralisation de la théorie de la radioactivité alpha. La probabilité de préformation de l'ion lourd, obtenue par un recouvrement des états nucléaires du noyau émis avec ceux du noyau parent, présente une grande difficulté de calcul pour $A_{2}>16$.

Une équivalence, au moins formelle, entre la probabilité de préformation de l'ion lourd (ou bien le facteur spectrométrique) et la pénétrabilité dans la région de la barrière de potentiel où il y a recouvrement des états nucléaires des fragments a été affirmée [7]. C'est la raison pour laquelle nous avons cherché à analyser les prédictions d'un modèle de fission pour des configurations de masses assymétriques extrêmes.

Pour le moment, la détection de ce type de désintégration a été limitée par certaines conditions :

i) A cause des difficultés expérimentales rencontrées au cours de l'identification des ions lourds noyés dans des flux très grands de particules alpha, le rapport de branchement doit être plus grand que $10^{-16}$ Pour réaliser de telles mesures, de nos jours, deux méthodes sont particulièrement utilisées : la déflection magnétique des particules alpha avec des spectromètres de grande efficacité et l'utilisation des détecteurs solides de traces qui sont pratiquement insensibles aux fortes doses alpha sous un certain seuil.

ii) Pour observer ce type de désintégration exotique, le noyau parent et le noyau descendant doivent avoir un temps de vie suffisamment long ; ils doivent donc se trouver à proximité de la ligne de stabilité.

iii) Pour des raisons énergétiques, le noyau descendant doit se trouver dans la région du ${ }^{208} \mathrm{~Pb}$ et le noyau émis doit être, de préférence, pair-pair. Cela explique l'excès de neutrons du fragment léger.

Pour analyser les résultats expérimentaux dans le cadre d'un modèle théorique, ces deux dernières conditions permettent d'approximer la configuration de scission par une paramétrisation de la forme nucléaire au moyen de deux sphères en contact. Cette approximation peut permettre le choix, comme on le verra par la suite, des conditions et valeurs initiales pour résoudre, par exemple, l'équation du mouvement.

\section{Paramétrisation de la forme nucléaire}

Pendant le processus de désintégration, la surface nucléaire est approximée par deux sphères reliées, sans discontinuités de premier ordre, par une surface obtenue par la rotation, autour de l'axe de symétrie, d'un arc de cercle [8], comme représenté sur la figure 1. La distance 


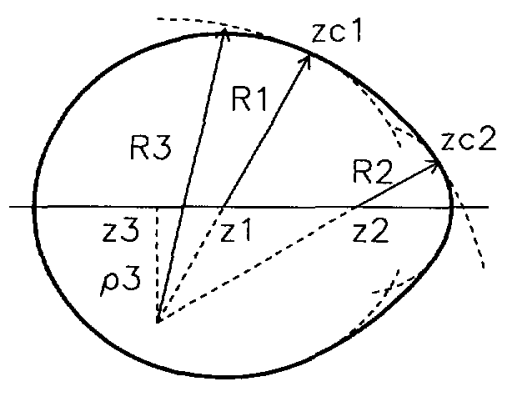

a) $S=-1$

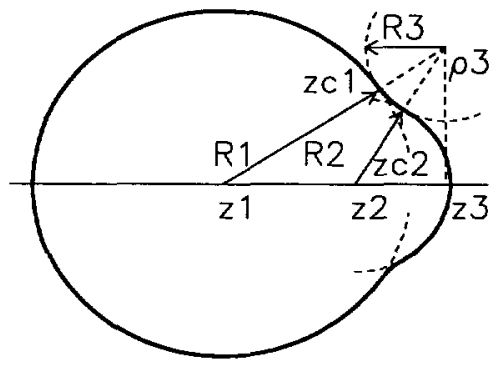

b) $S=+1$

Fig. 1. - Paramétrisation de la forme nucléaire.

[Parametrization of the nuclear shape.]

entre le centre de l'arc de cercle de la surface médiane et l'axe de symétrie est notée $\rho_{3}$. L'expression de cette dernière est choisie de telle sorte qu'elle prenne une valeur positive si les formes nucléaires obtenues présentent un col étroit ou, au contraire, une valeur négative si les configurations nucléaires sont gonflées( c'est-à-dire, présentent une forme proche de celle du diamant) dans la région médiane.

Le processus se produisant à très basse énergie, nous cherchons surtout à mettre en évidence le rôle joué par les trois coordonnées généralisées les plus pertinentes : élongation, striction (ou taille du col) et assymétrie de masse.

C'est la raison pour laquelle les coordonnées généralisées qui caractérisent les déformations de chaque fragment ne sont pas prises en considération. Pour simplifier les calculs, l'assymétrie de masse est donnée par le rayon du fragment léger, $R_{2}$, au lieu du conventionnel paramètre équivalent $\theta=\frac{A_{1}-A_{2}}{A_{0}}$, où $A_{0}, A_{1}$ et $A_{2}$ sont, respectivement, le nombre de masse du noyau parent, du noyau descendant et du fragment émis (nous garderons dorénavant ces indices de notation dans tout cet article). Le col est caractérisé par le rayon $R_{3}$ de l'arc de cercle, ou bien, alternativement, pour les cas où $R_{3}$ prend des valeurs élevées ou, quand les formes nucléaires sont gonflées, par le paramètre $C=S / R_{3}$ où $S=\operatorname{sgn}\left(\rho_{3}\right)$ : les formes en diamant sont obtenues pour $S=-1$ et l'existence d'un col pour $S=+1$. En imposant des valeurs pour les trois degrés de liberté les plus pertinents retenus et en respectant la condition de conservation de volume, on obtient des valeurs pour tous les paramètres qui contribuent à définir la forme nucléaire : $R_{1}$ qui représente le rayon du noyau descendant ; $z_{1}, z_{2}, z_{3}$ qui représentent les positions, sur l'axe de symétrie $\mathrm{O} z$, des centres des sphères de rayons $R_{1}$ et $R_{2}$, et de l'arc de cercle de rayon $R_{3}$, et $z_{c_{1}}$ et $z_{c_{2}}$ qui représentent les positions, sur l'axe $0 z$, des surfaces de séparation entre la surface médiane et les deux fragments. Du fait de la symétrie axiale, l'équation de la surface en coordonnées cylindriques est donnée, pour les trois régions considérées, par :

$$
\begin{aligned}
& \rho_{s}=\sqrt{R_{1}^{2}-\left(z-z_{1}\right)^{2}}, \quad z \leq z_{c_{1}} \\
& \rho_{s}=\rho_{3}-S \sqrt{R_{3}^{2}-\left(z-z_{3}\right)^{2}}, \quad z_{c_{1}} \leq z \leq z_{c_{2}} \\
& \rho_{s}=\sqrt{R_{2}^{2}-\left(z-z_{2}\right)^{2}}, \quad z>z_{c_{2}}
\end{aligned}
$$

Quand $S=\operatorname{sgn}\left(\rho_{3}\right)$ prend la valeur -1 , le volume $V_{2}$ du fragment émis est toujours calculé en 
intégrant sur l'intervalle $\left[z_{c_{2}}, z_{2}+R_{2}\right]$. Pour $S=+1, V_{2}$ est calculé dans l'intervalle $\left[z_{c_{2}}, z_{2}+R_{2}\right]$ dans le cas où $z_{c_{2}} \leq z_{3}$ et dans l'intervalle $\left[z_{3}, z_{2}+R_{2}\right]$ dans le cas où $z_{c_{2}}>z_{3}$.

Le volume du noyau descendant est alors : $V_{1}=V_{0}-V_{2}$.

Pendant la déformation, le volume total est conservé : $V_{0}=\frac{4 \pi}{3} r_{0} A_{0}^{\frac{1}{3}}$.

La valeur de la constante du rayon nucléaire utilisée dans ce travail est: $r_{0}=1,16 \mathrm{fm}$.

Les cas particuliers de deux sphères en intersection ou, au contraire, disjointes, sont obtenus pour $R_{3}=0$.

\section{Le tenseur d'inertie nucléaire}

L'énergie de déformation rend compte des forces généralisées qui agissent sur un noyau caractérisé par une paramétrisation donnée. Pour pouvoir décrire le processus de fission, il faut savoir également comment le noyau va réagir à ces forces. Cette information se trouve contenue dans le tenseur $B$ des coefficients d'inertie. Celui-ci rend compte de la dépendence de l'énergie cinétique du système nucléaire à l'égard des formes possibles du noyau dans le processus de désintégration :

$$
T=\frac{1}{2} \sum_{\imath, \jmath=1}^{n} B_{\imath \jmath} \dot{\beta}_{\imath} \dot{\beta}_{\jmath}
$$

Les paramètres $\beta_{\imath}$ et $\beta_{3}$ représentent les coordonnées généralisées. Le tenseur d'inertie $B$ est symétrique ; donc, pour les $n$ degrés de liberté, on obtient $n(n+1) / 2$ composantes indépendantes. Une méthode très connue de calcul du tenseur des masses effectives est l'approximation Werner-Wheeler. Dans cette approximation, on suppose que ce tenseur est une mesure du mouvement collectif et on cherche à le déterminer dans le cadre d'un modèle hydrodynamique pour un fluide incompressible, sans viscosité et irrotationel. En effectuant les calculs dans le système de coordonnées cylindriques, cette approximation revient à dire que la dérivée temporelle de $z$ pour déplacer un élément de masse nucléaire est indépendante de $\rho$ et que la dérivée temporelle de $\rho$ dépend linéairement de $\rho$ :

$$
\begin{aligned}
& \dot{z}=\sum_{\imath=1}^{n} A_{\imath}(z ; \beta) \dot{\beta}_{\imath} \\
& \dot{\rho}=\frac{\rho}{\rho_{s}} \sum_{\imath=1}^{n} B_{\imath}(z ; \beta) \beta_{\imath}
\end{aligned}
$$

où $\rho_{s}$ est la valeur de la coordonnée $\rho$ sur la surface nucléaire. Dans notre cas, $n=3$. Finalement, on obtient l'expression suivante pour les composantes du tenseur d'inertie :

$$
B_{\imath \jmath}=\pi \sigma_{m} \int_{z_{\min }}^{z_{\max }} \rho_{s}^{2}\left(A_{\imath} A_{\jmath}+\frac{1}{2} B_{\imath} B_{\jmath}\right) \mathrm{d} z+B_{\imath \jmath}^{\text {carr }}
$$

$\sigma_{m}$ étant la densité nucléaire. Les fonctions $A$ et $B$ résultent des hypothèses de l'approximation Werner-Wheeler et des conditions aux frontières qui imposent l'annulation de la composante normale de la vitesse sur la surface nucléaire. Ces fonctions ont les expressions suivantes :

$$
A_{11}(z ; \beta)=-\frac{1}{\rho_{s}^{2}} \frac{\partial}{\partial \beta_{\imath}} \int_{z_{\min }}^{z} \rho_{s}^{2} \mathrm{~d} x
$$




$$
\begin{aligned}
A_{\imath \mathrm{r}}(z ; \beta) & =-\frac{1}{\rho_{s}^{2}} \frac{\partial}{\partial \beta_{\imath}} \int_{z}^{z_{\max }} \rho_{s}^{2} \mathrm{~d} x \\
B_{\imath}(z ; \beta) & =-\frac{1}{2} \rho_{s} \frac{\partial A_{\imath}}{\partial z}
\end{aligned}
$$

où $z_{\min }, z_{\max }$ sont les valeurs des positions des extrêmités du noyau sur l'axe de symétrie. Les indices $l$ et $r$ signifient que les fonctions sont calculées, respectivement, pour la partie gauche et pour la partie droite du noyau.

Enfin, le dernier terme :

$$
B_{\imath \jmath}^{\text {corr }}=-\left(\pi^{2} \sigma_{m} / V\right) \int_{z_{\min }}^{z_{\max }} \rho_{s}^{2} A_{\imath} \mathrm{d} z \int_{z_{\min }}^{z_{\max }} \rho_{s}^{2} A_{\jmath} \mathrm{d} z
$$

représente une correction dûe au mouvement du centre de masse qui assure l'obtention d'une masse réduite correcte pour une configuration du système nucléaire réalisée par deux sphères disjointes ou en contact. Ce terme est non nul si l'origine de l'axe de symétrie $\mathrm{O} z$ ne coincide pas avec le centre de masse du système nucléaire.

Dans un espace multidimensionnel des configurations du système nucléaire, quand la forme nucléaire peut être décrite au moyen d'un jeu de $n$ paramètres indépendants, il est possible de réduire le problème multidimensionnel en un problème unidimensionnel et d'obtenir l'inertie scalaire $B(q)$ le long de la trajectoire dynamique, pour un chemin donné, de manière paramétrique, par les équations $\beta_{\imath}=\beta_{\imath}(q)$, au moyen de la relation suivante :

$$
B(q)=\sum_{\imath, j=1}^{n} B_{\imath \jmath}(\beta) \frac{\partial \beta_{\imath}}{\partial q} \frac{\partial \beta_{\jmath}}{\partial q}
$$

Ici, $q$ représente une variable indépendante (par exemple, l'élongation $R$ dans notre cas) par rapport à laquelle on veut déterminer le comportement des coordonnées généralisées.

Les dérivées $\partial R_{1} / \partial \beta_{\imath}$ et $\partial R_{2} / \partial \beta_{\imath}$ nécessaires au calcul des composantes du tenseur d'inertie ont été déterminées numériquement et l'intégration (5) a été effectuée au moyen des quadratures Gauss-Legendre.

Les effets de viscosité, au cours de la désintégration d'un système froid, pour lequel la vitesse de réarrangement des nucléons dans le champ moyen est beaucoup plus rapide que le taux de variation des coordonnées généralisées spécifiées, sont négligeables, en comparaison avec ce que l'on observe au cours du mécanisme habituel de fission globale. Cette hypothèse est justifiée par la valeur expérimentale de l'énergie cinétique des fragments, très proche de la chaleur de désintégration $Q$. Par conséquent, au cours de ce travail, pour ce type de processus exempt d'effets dissipatifs, on ne prend pas en considération le tenseur des forces de friction.

\section{Energie potentielle de déformation}

Pour le calcul de l'énergie de déformation, nous présentons ci-après le modèle de la goutte liquide pour une interaction nucléaire du type Yukawa-plus-exponentielle [9] dans le cadre du formalisme existant pour les systèmes binaires avec des densités de charge différentes, où un autre degré de liberté est introduit, c'est-à-dire, l'asymétrie de charge. La densité de charge du système est maintenue constante au cours des phases initiales du processus de désintégration, alors qu'au cours des dernières étapes de celui-ci la densité de charge de chaque fragment varie linéairement en fonction de l'élongation $R$ jusqu'à la valeur finale dans la voie de sortie. Un tel comportement est suggéré par le processus rapide d'équilibre de charge observé 
expérimentalement [10]. L'energie électrostatique est donnée par :

$$
E_{c}=\frac{1}{2} \int_{\infty} \int_{\infty} \frac{\rho_{e}\left(\mathbf{r}_{1}\right) \rho_{e}\left(\mathbf{r}_{2}\right)}{r_{12}} \mathrm{~d}^{3} r_{1} \mathrm{~d}^{3} r_{2}
$$

avec $r_{12}=\left|\mathbf{r}_{1}-\mathbf{r}_{2}\right|$. Si on considère que, pendant la déformation du système fissionnable, la densité de charge de chaque fragment naissant a une valeur constante dans chaque volume, pour les coordonnées généralisées fixées, l'intégrale ci-dessus peut être séparée en trois contributions :

$$
E_{c}=\frac{1}{2} \rho_{e_{1}}^{2} \int_{V_{1}} \mathrm{~d}^{3} r_{1} \int_{V_{1}} \frac{\mathrm{d}^{3} r_{2}}{r_{12}}+\rho_{e_{1}} \rho_{e_{2}} \int_{V_{1}} \int_{V_{2}} \frac{\mathrm{d}^{3} r_{1} \mathrm{~d}^{3} r_{2}}{r_{12}}+\frac{1}{2} \rho_{e_{2}}^{2} \int_{V_{2}} \mathrm{~d}^{3} r_{1} \int_{V_{2}} \frac{\mathrm{d}^{3} r_{2}}{r_{12}}
$$

où le premier et le dernier termes représentent les énergies propres des fragments, tandis que le second terme exprime l'énergie d'interaction.

Pendant le processus de désintégration, la charge est conservée :

$$
\rho_{e_{1}} V_{1}+\rho_{e_{2}} V_{2}=Z_{0}
$$

Pour déterminer l'énergie de surface $E_{Y}$, on utilise une formulation du type Yukawa-plusexponentielle :

$$
E_{Y}=-\frac{a_{2}}{8 \pi^{2} r_{0}^{2} a^{4}} \int_{V} \int_{V}\left(\frac{r_{12}}{a}-2\right) \frac{\exp \left(-\frac{r_{12}}{a}\right)}{\frac{r_{12}}{a}} \mathrm{~d}^{3} r_{1} \mathrm{~d}^{3} r_{2}
$$

avec une dépendence selon l'isospin :

$$
a_{2 \imath}=a_{s}\left(1-\kappa_{s} I_{\imath}\right)^{2}, \quad i=0,1,2
$$

Pour les systèmes binaires avec différentes densités de charge, on obtient :

$$
\begin{aligned}
E_{Y}= & -\frac{a_{21}}{8 \pi^{2} r_{0}^{2} a^{4}} \int_{V_{1}} \mathrm{~d}^{3} r_{1} \int_{V_{1}}\left(\frac{r_{12}}{a}-2\right) \frac{\exp \left(-\frac{r_{12}}{a}\right)}{\frac{r_{12}}{a}} \mathrm{~d}^{3} r_{2} \\
& -\frac{2 \sqrt{a_{21} a_{22}}}{8 \pi^{2} r_{0}^{2} a^{4}} \int_{V_{1}} \mathrm{~d}^{3} r_{1} \int_{V_{2}}\left(\frac{r_{12}}{a}-2\right) \frac{\exp \left(-\frac{r_{12}}{a}\right)}{\frac{r_{12}}{a}} \mathrm{~d}^{3} r_{2} \\
& -\frac{a_{22}}{8 \pi^{2} r_{0}^{2} a^{4}} \int_{V_{2}} \mathrm{~d}^{3} r_{1} \int_{V_{2}}\left(\frac{r_{12}}{a}-2\right) \frac{\exp \left(-\frac{r_{12}}{a}\right)}{\frac{r_{12}}{a}} \mathrm{~d}^{3} r_{2}
\end{aligned}
$$

avec une dépendence selon l'isospin obtenue avec les paramètres ajustables $a_{s}$ et $\kappa_{s}$. Les intégrations ont été obtenues par des quadratures Gauss-Legendre après avoir réduit analytiquement les intégrales sextuples à des intégrales doubles et triples, respectivement, pour l'énergie coulombienne et de surface, comme indiqué dans l'appendice $\mathrm{A}$.

Pour les systèmes assymétriques, on doit aussi ajouter une contribution à l'énergie totale, dûe à l'interaction de symétrisation qui intervient dans l'énergie de volume :

$$
E_{V}=E_{V_{1}}+E_{V_{2}}-E_{V_{0}} \neq 0
$$


avec

$$
\begin{array}{r}
E_{V_{\imath}}=-a_{1 \imath} A_{\imath}, \quad i=0,1,2 \\
a_{1 i}=a_{V}\left(1-\kappa_{V} I_{\imath}\right)^{2} .
\end{array}
$$

Les valeurs des paramètres ajustables sont :

$$
\begin{aligned}
a_{s} & =21,13 \mathrm{MeV} \\
\kappa_{s} & =2,3 \\
a & =0,68 \mathrm{fm} \\
a_{V} & =15,9937 \mathrm{MeV}
\end{aligned}
$$

et

$$
\kappa_{V}=1,927
$$

Enfin, on ajoute une correction phénoménologique en vue d'obtenir une valeur correcte de la chaleur de réaction.

$$
E_{\mathrm{cor}}(R)=\left(Q_{\mathrm{t}}-Q_{\mathrm{exp}}\right) \frac{V_{2}(R)}{V_{2 \mathrm{f}}}
$$

où $Q_{\mathrm{t}}$ et $Q_{\text {exp }}$ représentent, respectivement, les valeurs théorique et expérimentale de la chaleur de réaction.

Au début du processus de désintégration nucléaire, le volume $V_{2}$ du fragment léger est égal à zéro tandis que, au point de scission, il atteint sa valeur finale $V_{2 \mathrm{f}}$.

Cette correction est une approximation pour les effets de couches, d'appariement et de toutes contributions, autres que les premiers trois termes, que ce modèle simple ne prend pas en compte.

\section{Trajectoires de moindre action}

Pour l'étude de ces trois modes de désintégration (fission froide, émission spontanée d'ion lourd, désintégration alpha) dans un mode unitaire, on a déterminé des trajectoires optimales pour la pénétration de la barrière de potentiel multidimensionnelle en utilisant le principe de moindre action.

La pénétrabilité est calculée en utilisant l'approximation semi-classique Wentzel-KramersBrillouin (WKB) [11] :

$$
K=\frac{2}{\hbar} \int_{q_{1}}^{q_{2}} \sqrt{2 B(q, \beta, \dot{\beta})\left[E(q, \beta)-Q_{\exp }\right]} \mathrm{d} q=\frac{2}{\hbar} \int_{q_{1}}^{q_{2}} F(q, \beta, \dot{\beta}) \mathrm{d} q
$$

où $q_{1}$ et $q_{2}$ sont des points de rebroussement, où $B$ est l'inertie le long de la trajectoire dans la direction de la désintégration, où $\left(E(q, \beta)-Q_{\text {exp }}\right)$ représente l'énergie de déformation qu'on notera, dorénavant, $E$, pour la simplicité des calculs, et où $\beta$ représente l'ensemble des coordonnées généralisées.

Cette intégrale est calculée le long d'une trajectoire de moindre action pour une énergie donnée, qui relie la région d'énergies positives du puits de potentiel de l'état fondamental et le point de sortie de la barrière multidimensionnelle. Donc, le calcul de la pénétrabilité de la 
barrière de potentiel multidimensionnelle revient à trouver une trajectoire parmi le nombre infini de trajectoires possibles, qui corresponde à la plus petite valeur de l'intégrale d'action calculée dans le domaine, classiquement interdit, qui réunit les deux points $q_{1}$ et $q_{2}$ et pour toute la plage de variation de ces deux derniers points. Dans ce cas, le système peut évoluer vers des barrières énergétiques plus élevées si le paramètre d'inertie devient plus petit.

En général, du point de vue mathématique, on peut démontrer que, dans le cas où l'on connait les deux points à la frontière $\beta\left(q_{1}\right)$ et $\beta\left(q_{2}\right)$, une trajectoire, pour laquelle on obtient un extrêmum local, est obtenue par résolution du système des $n$ équations d'Euler-Lagrange :

$$
\frac{\partial F}{\partial \beta_{\imath}}-\frac{\mathrm{d}}{\mathrm{d} q}\left(\frac{\partial F}{\partial \dot{\beta}_{\imath}}\right)=0, \quad i=1, . . n
$$

Dans le cas analysé ici, on peut paramétriser la forme nucléaire, pour la configuration du point de scission, par deux sphères en contact. Cela permet de séparer la pénétrabilité en un produit de pénétrabilités :

$$
P=P_{o v} P_{s}=\exp [-K]=\exp \left[-K_{o v}-K_{s}\right]
$$

où $P_{o v}$ est la pénétrabilité relative au système dans l'état de prescission, tandis que $P_{s}$ a trait aux deux fragments séparés. $K_{o v}$ et $K_{s}$ représentent les valeurs des intégrales d'action pour les intervalles mentionnés. Cependant, pour un système formé de deux sphères complètement séparées, le col ne joue aucun rôle et l'assymétrie de masse est fixée. Donc, $P_{s}$ dépend uniquement de la distance entre les centres et peut être calculé très simplement sans minimisation.

Pour la région de recouvrement des deux fragments, où les énergies de déformation et les composantes inertielles sont obtenues par intégration numérique, il faut trouver une trajectoire permettant d'obtenir la valeur minimale de la relation fonctionnelle :

$$
K_{\text {ov }}=\frac{2}{\hbar} \int_{R_{2}}^{R_{s}} \sqrt{2 B\left(R, C, R_{2}, \frac{\partial C}{\partial R}, \frac{\partial R_{2}}{\partial R}\right) E\left(R, C, R_{2}\right)} \mathrm{d} R=\frac{2}{\hbar} \int_{R_{\imath}}^{R_{s}} F \mathrm{~d} R
$$

où les limites géométriques sont:

$$
\begin{aligned}
& R_{\mathrm{z}}=r_{0}\left(A_{0}^{1 / 3}-A_{2}^{1 / 3}\right) \\
& R_{s}=r_{0}\left(A_{1}^{1 / 3}+A_{2}^{1 / 3}\right)
\end{aligned}
$$

qui représentent, respectivement, la situation où la sphère de rayon $R_{2}$ commence à sortir du noyau initial et la configuration de scission. Pour simplifier les calculs, on a choisi comme paramètre d'intégration la coordonnée généralisée qui définit l'élongation.

Dans ce cas, on obtient le système d'équations d'Euler - Lagrange suivant :

$$
\begin{aligned}
& \frac{\partial F}{\partial Y}=\frac{\mathrm{d}}{\mathrm{d} R}\left(\frac{\partial F}{\partial \dot{Y}}\right) \\
& \frac{\partial F}{\partial X}=\frac{\mathrm{d}}{\mathrm{d} R}\left(\frac{\partial F}{\partial \dot{X}}\right)
\end{aligned}
$$

où $X=R_{2}$ et où on choisit $Y=R_{3}$ pour de faibles valeurs (plus petite que $1 \mathrm{fm}$ dans le programme numérique) du rayon qui caractérise le col et où on préfère, pour les autres valeurs de ce rayon, $Y=C$. On obtient un système de deux équations différentielles couplées du deuxième ordre :

$$
\begin{aligned}
& D_{X Y} \ddot{X}+D_{X Y_{3}} \dot{X}^{3}+D_{X Y_{2}} \dot{X}^{2}+D_{X Y_{1}} \dot{X}+D_{X Y_{0}}=0 \\
& D_{Y X} \ddot{Y}+D_{Y X_{3}} \dot{Y}^{3}+D_{Y X_{2}} \dot{Y}^{2}+D_{Y X_{1}} \dot{Y}+D_{Y X_{0}}=0
\end{aligned}
$$


où les dérivées $\dot{X}, \dot{Y}, \ddot{X}, \ddot{Y}$ sont calculées relativement à $R$ et où les coefficients $D$ sont des expressions mathématiques qui contiennent des dérivées partielles par rapport à $X$ et à $Y$. Ces coefficients sont symétriques en $X$ et $Y$ :

$$
\begin{aligned}
D_{X Y} & =2 E\left(A_{X Y} C_{X Y}-F_{X Y}^{2}\right) \\
D_{X Y_{3}} & =-2 E C_{X Y} \frac{\partial F_{X Y}}{\partial X}+C_{X Y}\left(C_{X Y} \frac{\partial E}{\partial R}-F_{X Y} \frac{\partial E}{\partial X}\right) \\
& +E\left(C_{X Y} \frac{\partial C_{X Y}}{\partial R}+F_{X Y} \frac{\partial C_{X Y}}{\partial X}\right) \\
D_{X Y_{2}} & =3 F_{X Y}\left(C_{X Y} \frac{\partial E}{\partial R}+E \frac{\partial C_{X Y}}{\partial R}\right)-\left(2 F_{X Y}^{2}+A_{X Y} C_{X Y}\right) \frac{\partial E}{\partial X} \\
& -2 E\left(C_{X Y} \frac{\partial A_{X Y}}{\partial X}+F_{X Y} \frac{\partial F_{X Y}}{\partial X}\right)+A_{X Y} E \frac{\partial C_{X Y}}{\partial X} \\
D_{X Y_{1}} & =-3 F_{X Y}\left(A_{X Y} \frac{\partial E}{\partial X}+E \frac{\partial A_{X Y}}{\partial X}\right)+\left(2 F_{X Y}^{2}+A_{X Y} C_{X Y}\right) \frac{\partial E}{\partial R} \\
& +2 E\left(A_{X Y} \frac{\partial C_{X Y}}{\partial R}+F_{X Y} \frac{\partial F_{X Y}}{\partial R}\right)-E C_{X Y} \frac{\partial A_{X Y}}{\partial R} \\
D_{X Y_{0}} & =2 E A_{X Y} \frac{\partial F_{X Y}}{\partial R}+A_{X Y}\left(F_{X Y} \frac{\partial E}{\partial R}-A_{X Y} \frac{\partial E}{\partial X}\right)-E\left(A_{X Y} \frac{\partial A_{X Y}}{\partial X}+F_{X Y} \frac{\partial A_{X Y}}{\partial R}\right)
\end{aligned}
$$

où

$$
\begin{aligned}
& A_{X Y}=B_{R R}+2 B_{R Y} \dot{Y}+B_{Y Y} \dot{Y}^{2} \\
& F_{X Y}=B_{R X}+B_{X Y} \dot{Y} \\
& C_{X Y}=B_{X X}
\end{aligned}
$$

Ainsi que nous l'avons vu dans les sections précédentes, les termes $B$ sont les coefficients d'inertie calculés par rapport aux coordonnées généralisées $X$ et $Y$ et $E$ est l'énergie de déformation.

Il est plus commode de résoudre le système d'équations (26) ci-dessus sous la forme suivante (nouvelle expression de la première équation seulement) :

$$
\begin{aligned}
T_{1 X Y} \ddot{X} & +T_{2 X Y} \ddot{X} \dot{Y}+T_{3 X Y} \ddot{X} \dot{Y}^{2}+T_{4 X Y} \dot{X}^{3}+T_{5 X Y} \dot{X}^{3} \dot{Y}+T_{6 X Y} \dot{X}^{2}+T_{7 X Y} \dot{X}^{2} \dot{Y} \\
& +T_{8 X Y} \dot{X}^{2} \dot{Y}^{2}+T_{9 X Y} \dot{X}+T_{10 X Y} \dot{X} \dot{Y}+T_{11 X Y} \dot{X}^{2}+T_{12 X Y} \dot{X} \dot{Y}^{3}+T_{13 X Y} \dot{X} \ddot{Y} \\
& +T_{14 X Y} \dot{X} \dot{Y}+T_{15 X Y}+T_{16 X Y} \dot{Y}+T_{17 X Y} \dot{Y}^{2}+T_{18 X Y} \dot{Y}^{3}+T_{19 X Y} \ddot{Y}+T_{20 X Y} \ddot{Y} \dot{Y} \\
& +T_{21 X Y} \dot{Y}^{4}=0
\end{aligned}
$$

Si les indices $X$ et $Y$, qui dénotent les coordonnées généralisées, sont inversés, on obtient la deuxième équation du système d'équations (26).

Les coefficients $T_{2 X Y}(\mathrm{i}=1, \ldots, 21)$ sont donnés dans l'appendice $\mathrm{B}$.

Pour résoudre l'équation (28), on a approximé les dérivées de premier et deuxième ordre par la méthode des différences finies :

$$
\begin{aligned}
\frac{\partial^{2} S}{\partial R^{2}} & =\frac{S_{\jmath+1}-2 S_{\jmath}+S_{\jmath-1}}{h^{2}} \\
\frac{\partial S}{\partial R} & =\frac{S_{3+1}-S_{j-1}}{h}, \quad j=1,2, . . m
\end{aligned}
$$

où $S$ peut être $X$ ou $Y$. 
On obtient un système de $2 m$ équations couplées avec $j=1,2, \ldots m$ et $2(m+2)$ quantités inconnues $X_{0}, X_{1}, \ldots X_{m+1}, Y_{0}, Y_{1}, . . Y_{m+1}$; l'intervalle de variation qui nous intéresse, $\left[R_{i}, R_{s}\right]$, étant divisé uniformément avec un incrément $h=\left(R_{s}-R_{\imath}\right) /(m+1)$.

Pour chaque point de l'axe $z$, donné par la relation $R_{j}=R_{s}-j h$, on obtient un système de deux équations :

$$
\begin{aligned}
& F_{1}\left(X_{\jmath+1}, X_{\jmath}, X_{\jmath-1}, Y_{\jmath+1}, Y_{\jmath}, Y_{\jmath-1}\right)=0 \\
& F_{2}\left(Y_{\jmath+1}, Y_{\jmath}, Y_{\jmath-1}, X_{\jmath+1}, X_{3}, X_{\jmath-1}\right)=0
\end{aligned}
$$

qui peut être résolu pas à pas.

Pour cela, on choisit différents jeux de valeurs initiales $X_{0}, X_{1}, Y_{0}$ et $Y_{1}$, qui doivent approximer la configuration donnée par deux sphères en contact. On résout les deux équations par la méthode itérative de Newton pour $j=1$ et on obtient $X_{2}$ et $Y_{2}$. On recommence ensuite pour $j=2$. Et ainsi de suite pour les autres valeurs de $j$.

L'avantage de cette méthode est que, pour chaque pas, on calcule une seule fois les valeurs des coefficients d'inertie et de l'énergie potentielle ; ce qui conduit à une économie du temps de calcul effectif. Au bout de plusieurs itérations, qui balaient le domaine des valeurs initiales qui approximent la configuration de scission, on retient les meilleures trajectoires. Pour la discrétisation de l'élongation $R$, on a choisi le nombre de pas de calcul égal à $m=1000$.

\section{Résultats}

Les trajectoires ont été calculées pour trois types de désintégrations nucléaires pour le même noyau parent ${ }^{238} \mathrm{Pu}$ : radioactivité alpha, émission du noyau ${ }^{32} \mathrm{Si}$ et fission froide avec, pour noyau léger, le noyau ${ }^{104} \mathrm{Mo}$. Bien que ce modèle ne soit pas approprié aux calculs de la radioactivité alpha, ces calculs ont été éffectués en vue d'une comparaison formelle avec l'expérience. Le comportement des trajectoires est conforme aux résultats déjà publiés [12] dans le cas du noyau parent ${ }^{234} \mathrm{U}$. Pour les trajectoires de moindre action de ces trois processus nucléaires, les coordonnées généralisées qui représentent la taille du col $\left(C\right.$ ou $\left.R_{3}\right)$ et l'assymétrie de masse $\left(R_{2}\right)$ sont représenteés sur les figures $2-4$, en fonction de l'élongation normalisée par rapport au domaine de recouvrement, intervalle où nous avons réalisé nos minimisations.

La région des élongations faibles n'est pas intéressante pour ce type de calculs, car les valeurs de l'énergie de déformation et des coefficients d'inertie sont très faibles. Par conséquent, les contributions qui proviennent de cette région sont négligeables par rapport aux valeurs totales des intégrales.

En analysant les calculs, nous avons constaté que, même pour l'intégrale $K_{\text {ov }}$, plus de 75 pour cent de la valeur totale provient de la moitié de l'intervalle des valeurs de l'élongation, pour le domaine de recouvrement, qui se trouve proche du point de scission. Dans le même temps, les valeurs très faibles des énergies de déformation et des inerties au début du processus de désintégration nucléaire conduisent également à une diminution de la précision des calculs au cours de la résolution des équations (30) déterminant les trajectoires optimales. Il est à remarquer, toutefois, que cette région de déformation n'est pas de grande importance pour le processus dynamique.

Dans le cas de la fission froide et de l'émission exotique, on peut observer, sur les figures 2 et 3, qu'au début du processus de désintégration nucléaire les formes en diamant sont privilégiées, les valeurs de $\mathrm{C}$ étant négatives.

Sur ces deux figures, deux différences existent cependant : premièrement, dans le cas de la fission, la taille du col commence à diminuer pour des valeurs plus grandes de l'élongation $(C \rightarrow \infty)$, presque au voisinage du point de scission, si on compare ce comportement par 


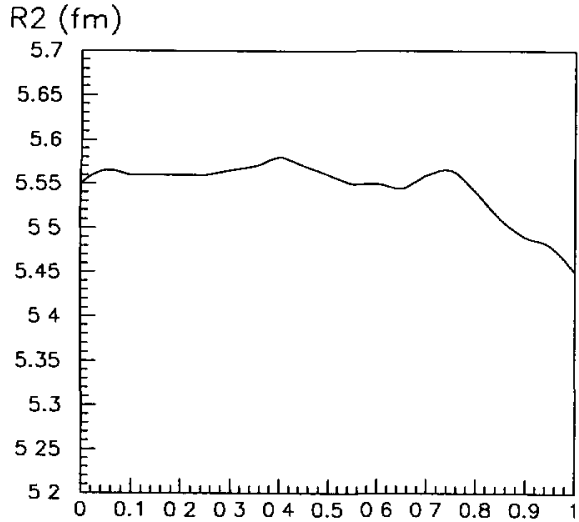

a)

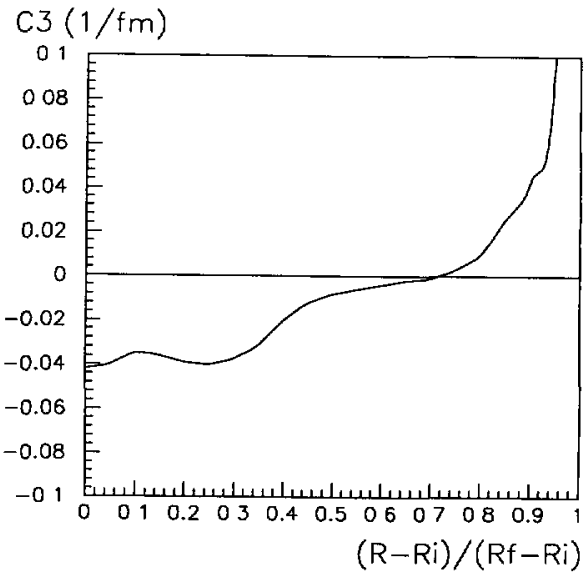

b)

Fig. 2. - Trajectoire de moindre action pour la fission froide du ${ }^{238} \mathrm{Pu}$, avec pour fragment léger, le ${ }^{104} \mathrm{Mo}$. En haut, on a représenté la variation de l'assymétrie de masse $R_{2}$ par rapport à l'élongation normalisée $\left(R-R_{3}\right) /\left(R_{f}-R_{2}\right)$, et, en bas, la variation du col $C$ par rapport au même paramètre.

[Variation of $R_{2}$ (upper part,a) and of $C_{3}$ (lower part,b) with the relative separation distance between centers, along the optimum fission path for cold fission with ${ }^{104} \mathrm{Mo}$ light fragment, of ${ }^{238} \mathrm{Pu}$.]

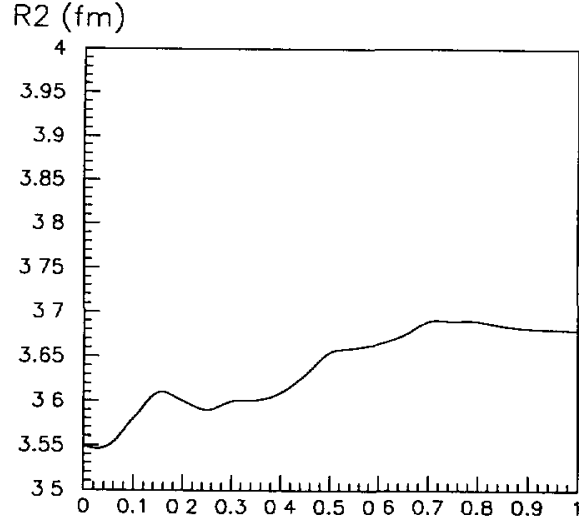

a)

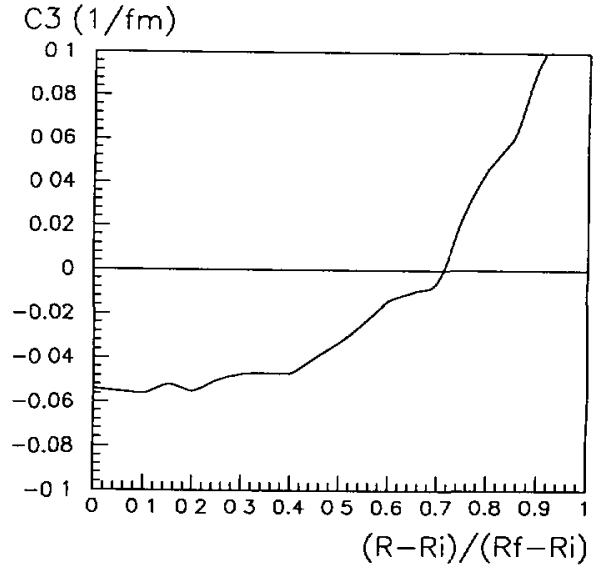

b)

Fig. 3. - Trajectoire de moindre action pour l'émission du fragment ${ }^{32} \mathrm{Si}$ par le ${ }^{238} \mathrm{Pu}$. En haut, on a représenté la variation de l'assymétrie de masse $R_{2}$ par rapport à l'élongation normalisée $\left(R-R_{\imath}\right) /\left(R_{f}-R_{\imath}\right)$, et, en bas, la variation du col $C$ par rapport au même paramètre.

[Variation of $R_{2}$ (upper part,a) and of $C_{3}$ (lower part,b) with the relative separation distance between centers, along the optimum fission path ${ }^{32} \mathrm{Si}$ radioactivity of ${ }^{238} \mathrm{Pu}$.]

rapport à l'évolution analogue dans le cas de l'émission d'ions lourds ; et, deuxièmement, pour la radioactivité avec émission du noyau ${ }^{32} \mathrm{Si}$, au début du processus, des formes plus compactes (sphéroïdales) sont privilégiées, c'est-à-dire, $C$ prend des valeurs négatives plus grandes pour ce mode de désintégration nucléaire que pour la fission froide. On obtient une situation complètement différente en ce qui concerne le comportement du col pour la radioactivité alpha ; car 


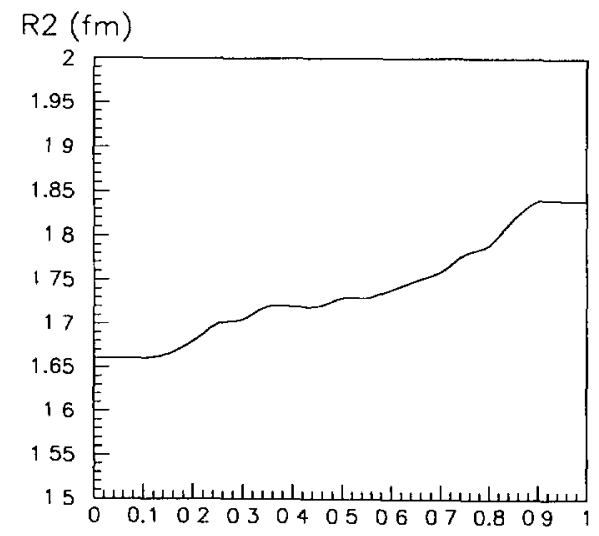

a)

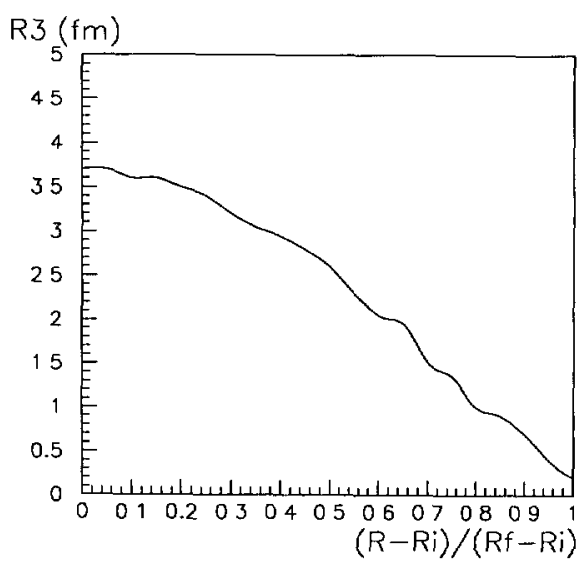

b)

Fig. 4. - Trajectoire de moindre action pour la désintégration alpha du ${ }^{238} \mathrm{Pu}$. En haut, on a représenté la variation de l'assymétrie de masse $R_{2}$ par rapport à l'élongation normalisée $\left(R-R_{i}\right) /\left(R_{f}-R_{\imath}\right)$, et, en bas, la variation du col $R_{3}$ par rapport au même paramètre.

[Variation of $R_{2}$ (upper part,a) and of $C_{3}$ (lower part,b) with the relative separation distance between centers, along the optimum fission path for $\alpha$-decay, of ${ }^{238} \mathrm{Pu}$.]

on a obtenu des valeurs de $R_{3}$ très faibles sur tout le domaine de recouvrement et des formes nucléaires physiquement acceptables seulement avec $S=1$.

Pour l'assymétrie de masse caractérisée par la coordonnée généralisée $R_{2}$, on peut observer que, dans le cas de la fission, on a une tendance de symétrisation au début du processus tandis que, pour l'émission exotique, l'assymétrie est plus prononcée. Pour la désintégration alpha, la variation de $R_{2}$ est très faible ; ce qui prouve, une fois de plus, la validité de la paramétrisation de la forme nucléaire par deux sphères qui se recouvrent partiellement, paramétrisation déjà utilisée pour ce type de processus nucléaire.

Les séquences de variation des formes nucléaires au cours de ces trois types de processus nucléaire, en partant depuis l'état fondamental jusqu'au point de scission, sont représentées sur la figure 5 .

Pour les temps de vie du noyau parent ${ }^{238} \mathrm{Pu}$, on a, d'après les références $[7,13,14], \log (T[\mathrm{~s}])=$ 9,6 pour la désintégration alpha et $\log (T[\mathrm{~s}])=25,3$ pour l'émission spontanée du noyau ${ }^{32} \mathrm{Si}$. Le modèle de fission conduit à l'estimation du temps de vie selon la formule :

$$
T=\frac{h \ln (2)}{2 E_{v}} \exp (K)
$$

où $E_{v}$ représente l'énergie de vibration du point zéro et exp $(\mathrm{K})$ nous donne la pénétrabilité totale dans la barrière multidimensionnelle.

Conformément à ce modèle, si on ne fait aucune hypothèse sur la valeur de $E_{v}$, le rapport des temps de vie relatifs à la désintégration alpha et à la radioactivité par émission du noyau ${ }^{32} \mathrm{Si}$ sera égal à :

$$
b_{\alpha / \mathrm{Si}}=\frac{\exp \left(K_{\alpha}\right)}{\exp \left(K_{\mathrm{Si}}\right)}
$$




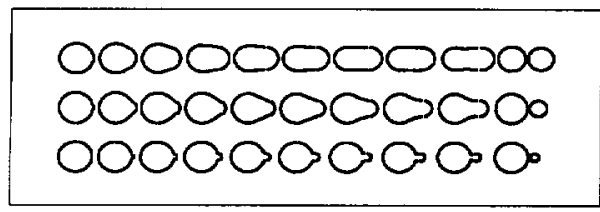

Fig. 5. - Formes nucléaires déterminées le long de la trajectoire de moindre action pour la fission froide avec, pour fragment léger, le ${ }^{104} \mathrm{Mo}$ (en haut), l' émission $\mathrm{du}^{32} \mathrm{Si}$ (au milieu) et la désintégration alpha (en bas) du même noyau parent, le ${ }^{238} \mathrm{Pu}$.

[Nuclear shapes along optimum fission paths for cold fission with ${ }^{104} \mathrm{Mo}$ light fragment (top), ${ }^{32} \mathrm{Si}$ radioactivity (middle) and $\alpha$-decay (bottom) of ${ }^{238} \mathrm{Pu}$.]

Les valeurs théoriques de l'intégrale d'action ont été obtenues par intégration numérique, après avoir interpolé les trajectoires au moyen des fonctions splines. Si on ne prend pas en considération les coordonnées généralisées caractérisant la taille du col et l'assymétrie de masse, on obtient une paramétrisation donnée par deux sphères qui se recouvrent, avec le rayon du fragment léger constant et égal à sa valeur finale. Dans ce cas, nous avons obtenu les résultats théoriques suivants : $K_{o v}=95,87 ; 45,6 ; 6,02$ et $K_{s}=53,12 ; 72,22 ; 69,75$, respectivement, pour la fission, l'émission d'ions lourds et la radioactivité alpha. Après minimisation dans la région de recouvrement, nous avons obtenu, pour la même séquence de processus de désintégrations nucléaires, $K_{o v}^{\text {optimal }}=71,35 ; 37,92 ; 5,11$. Ces résultats sont ce que l'on peut appeler des valeurs optimisées. Nous avons obtenu $b_{\alpha / \mathrm{Si}}^{\text {optimal }}=4,76 \times 10^{-16}$ pour les valeurs optimisées et $b_{\alpha / \mathrm{Si}}=5,5 \times 10^{-19}$ pour les valeurs non optimisées, c'est-à-dire lorsque l'on considère une paramétrisation de la forme nucléaire donnée par deux sphères qui se recouvrent, avec la coordonnée qui caractérise l'assymétrie de masse maintenue constante. La valeur expérimentale est $b_{\alpha / \mathrm{Si}}^{\exp }=2 \times 10^{-16}$ Ces comparaisons indiquent une amélioration de ce modèle après minimisation. Dans le même temps, ces calculs optimisés prédisent, selon cette procédure, un temps de vie, relatif à la fission froide avec, pour fragment léger, le noyau ${ }^{104} \mathrm{Mo}$, estimé par $\log (T[\mathrm{~s}])=31,1$.

\section{Remerciements}

Nous remercions chaleureusement $\mathrm{R}$. Bimbot et $\mathrm{F}$. Clapier pour le vif intérêt témoigné à ce travail, ainsi que pour l'aide constante qu'ils nous ont apportée et sans laquelle il n'aurait pu être mené à bien.

\section{Appendice A}

Connaissant l'énergie électrostatique d'une sphère uniformément chargée,

$$
E_{c_{0}}=\frac{3}{5} \frac{e^{2} Z^{2}}{r_{0} A^{\frac{1}{3}}}
$$

on peut obtenir les termes $B_{c}$ qui dépendent de la forme du noyau à partir de la relation (12) :

$$
\begin{aligned}
B_{c} & =\frac{E_{c}}{E_{c_{0}}} \\
& =\left(\frac{\rho_{e_{1}}}{\rho_{e_{0}}}\right)^{2} B_{c_{1}}+\frac{\rho_{e_{1}} \rho_{\varepsilon_{2}}}{\rho_{e_{0}}^{2}} B_{c_{12}}+\left(\frac{\rho_{e_{2}}}{\rho_{e_{0}}}\right)^{2} B_{c_{2}} .
\end{aligned}
$$


Conformément à la méthode Davies-Sierk [15], on peut écrire :

$$
\begin{aligned}
B_{c_{1}} & =b_{c} \int_{-1}^{x_{3}} \mathrm{~d} x \int_{-1}^{x_{3}} \mathrm{~d} x^{\prime} F\left(x, x^{\prime}\right) \\
B_{c_{12}} & =2 b_{c} \int_{-1}^{x_{3}} \mathrm{~d} x \int_{x_{3}}^{1} \mathrm{~d} x^{\prime} F\left(x, x^{\prime}\right) \\
B_{c_{2}} & =b_{c} \int_{x_{3}}^{1} \mathrm{~d} x \int_{x_{3}}^{1} \mathrm{~d} x^{\prime} F\left(x, x^{\prime}\right)
\end{aligned}
$$

où : $b_{c}=5 d^{5} /(8 \pi)$ et $d=\left(z_{\max }-z_{\min }\right) /\left(2 R_{0}\right) ; x_{3}$ est la position du plan de séparation des fragments sur l'axe $z$ en unités $2 d$ et prend des valeurs entre -1 et $1 ; z_{\min }$ et $z_{\max }$ sont les valeurs des positions des extrêmités du noyau sur l'axe $z$ de symétrie $z$. Ainsi :

$$
\begin{aligned}
F\left(x, x^{\prime}\right) & =\left\{y y_{1} \frac{K-2 D}{3}\right. \\
& \times\left[2\left(y^{2}+y_{1}^{2}\right)\left(x-x^{\prime}\right)^{2}+\frac{3}{2}\left(x-x^{\prime}\right)\left(\frac{\mathrm{d} y_{1}^{2}}{\mathrm{~d} x^{\prime}}-\frac{\mathrm{d} y^{2}}{\mathrm{~d} x}\right)\right] \\
& \left.+k\left\{\frac{y^{2} y_{1}^{2}}{3}+\left[y^{2}-\frac{1}{2}\left(x-x^{\prime}\right) \frac{\mathrm{d} y^{2}}{\mathrm{~d} x}\right]\left[y_{1}^{2}+\frac{1}{2}\left(x-x^{\prime}\right) \frac{\mathrm{d} y_{1}^{2}}{\mathrm{~d} x^{\prime}}\right]\right\}\right\} a_{\rho}^{-1}
\end{aligned}
$$

où $y_{1}=y\left(x^{\prime}\right)$ est l'équation de la surface nucléaire dans le domaine $(-1,1)$,

$$
\begin{aligned}
a_{\rho}^{2} & =\left(y+y_{1}\right)^{2}+\left(x-x^{\prime}\right)^{2} \\
k^{2} & =\frac{4 y y_{1}}{a_{\rho}^{2}} \\
\text { et } & \\
D & =\frac{K-K^{\prime}}{k^{2}}
\end{aligned}
$$

$K$ et $K^{\prime}$ sont des intégrales elliptiques du premier et deuxième ordre, respectivement :

$$
\begin{aligned}
K(k) & =\int_{0}^{\frac{\pi}{2}}\left(1-k^{2} \sin ^{2} t\right)^{-\frac{1}{2}} \mathrm{~d} t \\
K^{\prime}(k) & =\int_{0}^{\frac{\pi}{2}}\left(1-k^{2} \sin ^{2} t\right)^{\frac{1}{2}} \mathrm{~d} t
\end{aligned}
$$

Dans le cas particulier de l'interaction de deux sphères disjointes, on obtient :

$$
E_{c_{12}}=\frac{Z_{1} Z_{2} e^{2}}{R}
$$

La relation (14) donne, pour une sphère :

$$
E_{Y_{0}}=a_{20} A^{\frac{1}{3}}\left\{1-3\left(\frac{a}{R_{0}}\right)^{2}+\left(1+\frac{R_{0}}{a}\right)\left[2+\frac{3}{R_{0}}\left(1+\frac{a}{R_{0}}\right)\right] \exp \left(-2 \frac{R_{0}}{a}\right)\right\}
$$

ce qui est proportionnel à $A^{\frac{2}{3}}$, donc seulement à un terme de surface.

L'énergie relative est :

$$
\begin{aligned}
B_{Y} & =\frac{E_{Y}}{E_{Y 0}} \\
& =\frac{a_{21}}{a_{20}} B_{Y_{1}}+\frac{\sqrt{a_{21} a_{22}}}{a_{20}} B_{Y_{12}}+\frac{a_{22}}{a_{20}} B_{Y_{2}}
\end{aligned}
$$


où les quantités qui dépendent de la forme nucléaire sont :

$$
\begin{aligned}
B_{Y_{1}} & =-\frac{r_{0}}{2 a^{2}} \frac{a_{2} R_{0} A}{E_{y}^{0}}(2 d)^{4} \int_{-1}^{z_{3}} \int_{-1}^{z_{3}} \int_{0}^{1} F_{1}\left(x, x^{\prime}\right) F_{2}\left(x, x^{\prime}\right) Q_{Y}\left(x, x^{\prime}\right) \mathrm{d} x \mathrm{~d} x^{\prime} \mathrm{d} w \\
B_{Y_{12}} & =-2 \frac{r_{0}}{2 a^{2}} \frac{a_{2} R_{0} A}{E_{y}^{0}}(2 d)^{4} \int_{-1}^{z_{3}} \int_{z_{3}}^{1} \int_{0}^{1} F_{1}\left(x, x^{\prime}\right) F_{2}\left(x, x^{\prime}\right) Q_{Y}\left(x, x^{\prime}\right) \mathrm{d} x \mathrm{~d} x^{\prime} \mathrm{d} w \\
B_{Y_{2}} & =-\frac{r_{0}}{2 a^{2}} \frac{a_{2} R_{0} A}{E_{y}^{0}}(2 d)^{4} \int_{z_{3}}^{1} \int_{z_{3}}^{1} \int_{0}^{1} F_{1}\left(x, x^{\prime}\right) F_{2}\left(x, x^{\prime}\right) Q_{Y}\left(x, x^{\prime}\right) \mathrm{d} x \mathrm{~d} x^{\prime} \mathrm{d} w
\end{aligned}
$$

et

$$
\begin{aligned}
& Q_{Y}=\left\{\left[P^{\frac{1}{2}}\left(P^{\frac{1}{2}}+\frac{2 a}{R_{0} l}\right)+\frac{2 a^{2}}{\left(R_{0} l\right)^{2}}\right] \exp \left(-\frac{R_{0} l P^{\frac{1}{2}}}{a}\right)-\frac{2 a^{2}}{\left(R_{0} l\right)^{2}}\right\} / P^{2} \\
& F_{1}=y^{2}-y y_{1} \cos \varphi-0,5\left(x-x^{\prime}\right) \frac{\mathrm{d} y^{2}}{\mathrm{~d} x} \\
& F_{2}=y_{1}^{2}-y y_{1} \cos \varphi+0,5\left(x-x^{\prime}\right) \frac{\mathrm{d} y_{1}^{2}}{\mathrm{~d} x^{\prime}} \\
& P=\sqrt{y^{2}+y_{1}^{2}-2 y y_{1} \cos \varphi+\left(x-x^{\prime}\right)^{2}} \\
& \varphi=2 \pi w, \quad l=d
\end{aligned}
$$

Pour l'énergie d'interaction de deux sphères disjointes, on a les relations suivantes :

$$
\begin{aligned}
E_{Y_{12}} & =\left(\frac{a}{r_{0}}\right)^{2} \sqrt{a_{21} a_{22}}\left[g_{1} g_{2}\left(4+\frac{R}{a}\right)-g_{2} f_{1}-g_{1} f_{2}\right] \frac{\exp \left(-\frac{R}{a}\right)}{\frac{R}{a}} \\
g_{k} & =\frac{R_{k}}{a} \cosh \left(\frac{R_{k}}{a}\right)-\sinh \left(\frac{R_{k}}{a}\right), \quad k=1,2 \\
f_{k} & =\left(\frac{R_{k}}{a}\right)^{2} \sinh \left(\frac{R_{k}}{a}\right)
\end{aligned}
$$

\section{Appendice B}

Les coefficients $T_{\imath X Y}(i=1, \ldots, 21)$ de l'équation (28) :

$$
\begin{aligned}
T_{1 X Y} \ddot{X} & +T_{2 X Y} \ddot{X} \dot{Y}+T_{3 X Y} \ddot{X} \dot{Y}^{2}+T_{4 X Y} \dot{X}^{3}+T_{5 X Y} \dot{X}^{3} \dot{Y} \\
& +T_{6 X Y} \dot{X}^{2}+T_{7 X Y} \dot{X}^{2} \dot{Y}+T_{8 X Y} \dot{X}^{2} \dot{Y}^{2}+T_{9 X Y} \dot{X}+T_{10 X Y} \dot{X} \dot{Y} \\
& +T_{11 X Y} \dot{X} \dot{Y}^{2}+T_{12 X Y} \dot{X} \dot{Y}^{3}+T_{13 X Y} \dot{X} \ddot{Y}+T_{14 X Y} \dot{X} \dot{Y} \ddot{Y}+T_{15 X Y} \\
& +T_{16 X Y} \dot{Y}+T_{17 X Y} \dot{Y}^{2}+T_{18 X Y} \dot{Y}^{3}+T_{19 X Y} \ddot{Y}+T_{20 X Y} \ddot{Y} \dot{Y} \\
& +T_{21 X Y} \dot{Y}^{4}=0
\end{aligned}
$$

sont donnés par les formules :

$$
\begin{aligned}
& T_{1 X Y}=2 E\left(B_{R R} B_{X X}-B_{R X}^{2}\right) \\
& T_{2 X Y}=4 E\left(B_{R Y} B_{X X}-B_{R X} B_{Y X}\right) \\
& T_{3 X Y}=2 E\left(B_{Y Y} B_{X X}-B_{X Y}^{2}\right)
\end{aligned}
$$




$$
\begin{aligned}
& T_{4 X Y}=-2 E B_{X X} \frac{\partial B_{R X}}{\partial X}+B_{X X}^{2} \frac{\partial E}{\partial R}-B_{X X} B_{R X} \frac{\partial E}{\partial X} \\
& +E B_{X X} \frac{\partial B_{X X}}{\partial R}+E B_{R X} \frac{\partial B_{X X}}{\partial X} \\
& T_{5 X Y}=E B_{X Y} \frac{\partial B_{X X}}{\partial X}-2 E B_{X X} \frac{\partial B_{X Y}}{\partial X}-B_{X X} B_{X Y} \frac{\partial E}{\partial X} \\
& T_{6 X Y}=3 B_{R X} B_{X X} \frac{\partial E}{\partial R}+3 B_{R X} E \frac{\partial B_{X X}}{\partial R}-2 B_{R Y}^{2} \frac{\partial E}{\partial X} \\
& \text { - } B_{R R} B_{X X} \frac{\partial E}{\partial X}-2 E B_{X X} \frac{\partial B_{R R}}{\partial X}-2 E B_{R X} \frac{\partial B_{R X}}{\partial X}+E B_{R R} \frac{\partial B_{X X}}{\partial X} \\
& T_{7 X Y}=3 B_{X Y} B_{X X} \frac{\partial E}{\partial R}+3 B_{X Y} E \frac{\partial B_{X X}}{\partial R}-4 B_{R X} B_{X Y} \frac{\partial E}{\partial X} \\
& \text { - } 4 E B_{X X} \frac{\partial B_{R Y}}{\partial X}-2 E B_{X Y} \frac{\partial B_{R X}}{\partial X}-2 E B_{R X} \frac{\partial B_{X Y}}{\partial X} \\
& -2 B_{R Y} B_{X X} \frac{\partial E}{\partial X}+2 E B_{R Y} \frac{\partial B_{X X}}{\partial X} \\
& T_{8 X Y}=E B_{Y Y} \frac{\partial B_{X X}}{\partial X}-2 B_{X Y}^{2} \frac{\partial E}{\partial X}-B_{Y Y} B_{X X} \frac{\partial E}{\partial X}-2 E B_{X X} \frac{\partial B_{Y Y}}{\partial X}-2 E B_{X Y} \frac{\partial B_{X Y}}{\partial X} \\
& T_{9 X Y}=-3 B_{R X} B_{R R} \frac{\partial E}{\partial X}+3 E B_{R X} \frac{\partial B_{R R}}{\partial X}+2 B_{R X}^{2} \frac{\partial E}{\partial R} \\
& +B_{R R} B_{X X} \frac{\partial E}{\partial R}+2 E B_{R R} \frac{\partial B_{X X}}{\partial R}+2 E B_{R X} \frac{\partial B_{R X}}{\partial R}-E B_{X X} \frac{\partial B_{R R}}{\partial R} \\
& T_{10 X Y}=-6 B_{R X} B_{R Y} \frac{\partial E}{\partial X}+6 E B_{R X} \frac{\partial B_{R Y}}{\partial X}-3 B_{X Y} B_{R R} \frac{\partial E}{\partial X} \\
& +3 E B_{X Y} \frac{\partial B_{R R}}{\partial X}+4 B_{R X} B_{X Y} \frac{\partial E}{\partial R}+2 B_{R Y} B_{X X} \frac{\partial E}{\partial R} \\
& +4 E B_{R Y} \frac{\partial B_{X X}}{\partial R}+2 E B_{R X} \frac{\partial B_{X Y}}{\partial R}+2 E B_{X Y} \frac{\partial B_{R X}}{\partial R}-2 E B_{X X} \frac{\partial B_{R Y}}{\partial R}
\end{aligned}
$$

$$
\begin{aligned}
T_{11 X Y} & =-3 B_{R X} B_{Y Y} \frac{\partial E}{\partial X}+3 E B_{R X} \frac{\partial B_{Y Y}}{\partial X}-6 B_{X Y} B_{R Y} \frac{\partial E}{\partial X} \\
& +6 E B_{X Y} \frac{\partial B_{R Y}}{\partial X}+2 B_{X Y}^{2} \frac{\partial E}{\partial R}+B_{X X} B_{Y Y} \frac{\partial E}{\partial R} \\
& +2 E B_{Y Y} \frac{\partial B_{X X}}{\partial R}+2 E B_{X Y} \frac{\partial B_{X Y}}{\partial R}-E B_{X X} \frac{\partial B_{Y Y}}{\partial R} \\
T_{12 X Y} & =-3 B_{X Y} B_{Y Y} \frac{\partial E}{\partial X}+3 E B_{X Y} \frac{\partial B_{Y Y}}{\partial X} \\
T_{13 X Y} & =2 E B_{R X} B_{X Y}-2 E B_{X X} B_{R Y} \\
T_{14 X Y} & =2 E B_{X Y}^{2}-2 E B_{X X} B_{Y Y} \\
T_{15 X Y} & =2 E B_{R R} \frac{\partial B_{R X}}{\partial R}+B_{R R} B_{R X} \frac{\partial E}{\partial R}-B_{R R}^{2} \frac{\partial E}{\partial X}-E B_{R R} \frac{\partial B_{R R}}{\partial X}-E B_{R X} \frac{\partial B_{R R}}{\partial R} \\
T_{16 X Y} & =2 E B_{R R} \frac{\partial B_{X Y}}{\partial R}+4 E B_{R Y} \frac{\partial B_{R X}}{\partial R}+B_{R R} B_{X Y} \frac{\partial E}{\partial R} \\
& +2 B_{R X} B_{R Y} \frac{\partial E}{\partial R}-4 B_{R R} B_{R Y} \frac{\partial E}{\partial X}-2 E B_{R R} \frac{\partial B_{R Y}}{\partial X} \\
& -2 E B_{R Y} \frac{\partial B_{R R}}{\partial X}-2 E B_{R X} \frac{\partial B_{R Y}}{\partial R}-E B_{X Y} \frac{\partial B_{R R}}{\partial R}
\end{aligned}
$$




$$
\begin{aligned}
T_{17 X Y} & =4 E B_{R Y} \frac{\partial B_{X Y}}{\partial R}+2 E B_{Y Y} \frac{\partial B_{R X}}{\partial R}+2 B_{R Y} B_{X Y} \frac{\partial E}{\partial R} \\
& +B_{Y Y} B_{R X} \frac{\partial E}{\partial R}-2 B_{R R} B_{Y Y} \frac{\partial E}{\partial X}-4 B_{R Y}^{2} \frac{\partial E}{\partial X} \\
& -E B_{R R} \frac{\partial B_{Y Y}}{\partial X}-4 E B_{R Y} \frac{\partial B_{R Y}}{\partial X}-E B_{Y Y} \frac{\partial B_{R R}}{\partial X} \\
& -E B_{R X} \frac{\partial B_{Y Y}}{\partial R}-2 E B_{X Y} \frac{\partial B_{R Y}}{\partial R} \\
T_{18 X Y} & =2 E B_{Y Y} \frac{\partial B_{X Y}}{\partial R}+B_{X Y} B_{Y Y} \frac{\partial E}{\partial R}-4 B_{R Y} B_{Y Y} \frac{\partial E}{\partial X} \\
& -2 E B_{R Y} \frac{\partial B_{Y Y}}{\partial X}-2 E B_{Y Y} \frac{\partial B_{R Y}}{\partial X}-E B_{X Y} \frac{\partial B_{X Y}}{\partial R} \\
T_{19 X Y} & =2 E\left(B_{X Y} B_{R R}-B_{R X} B_{R Y}\right) \\
T_{20 X Y} & =2 E\left(B_{X Y} B_{R Y}-B_{R X} B_{Y Y}\right) \\
T_{21 X Y} & =-B_{Y Y}^{2} \frac{\partial E}{\partial X}-E B_{Y Y} \frac{\partial B_{Y Y}}{\partial X}
\end{aligned}
$$

Les expressions des coefficients $T_{\imath Y X}$ sont déduites des relations précédentes en interchangeant $\mathrm{X}$ et $\mathrm{Y}$.

\section{Bibliographie}

[1] Sandulescu A., Poenaru D.N. et Greiner W., New type of decay of heavy nuclei intermediate between fission and alpha-decay, Sov. J. Part. Nucl. 11 (1980) 528.

[2] Poenaru D.N. et Ivascu M., "Fission at very large mass and charge", Critical Phenomena in Heavy Ion Physics (Raduta A. et Stratan G., Eds., ICEFIZ, Bucarest, 1980) p. 743.

[3] Rose H.J. et Jones G.A., A new kind of natural radioactivity, Nat. 307 (1984) 245.

[4] Hourani E., "Carbone decay and fine structure", Nuclear Decay Modes (Poenaru D.N. et Greiner W., Eds.) à paraître ; Bonetti R. et Guglielmetti A., Nuclear Decay Modes (Poenaru D.N. et Greiner W., Eds.) à paraître.

[5] Brillard L., Elayi A.G., Hourani E., Hussonnois M., Le Du J.F., Rosier L.H. et Stab L., Mise en évidence d'une structure fine dans la radioactivité du ${ }^{14} \mathrm{C}$, Acad.Scr. Paris C.R. 309 (1989) 1105.

[6] Blendowske R. et Walliser H., Systematics of cluster-radioactivity-decay constants as suggested by microscopic calculations, Phys. Rev. Lett. 61 (1988) 1930.

[7] Poenaru D.N. et Greiner W., Rare decay modes by cluster emission from nuclei, J.Phys. G : Nucl. Part. Phys. 17 (1991) S 443.

[8] Depta K., Herrman R., Maruhn J.A. et Greiner W., Dynamics of Collective Phenomena (David P., Ed., World Scientific, Singapore, 1987)p. 29.

[9] Krappe H.J., Nix J.R. et Sierk A.J., Unified nuclear potential for heavy-ion elastic scattering, fusion, fission and ground state masses and deformations, Phys. Rev. C 20 (1979) 992.

[10] , Poenaru D.N., Mazilu D. et Ivascu M., Deformation energies for nuclei with different charge-tomass ratio, J. Phys. G 5 (1979) 1093.

[11] Brack M., Damgaard J., Jensen A., Pauli H.C., Strutinsky V.M. et Wong G.I., Funny Hills: the shell correction approach to nuclear shell effects and its applications to the fission process, Rev. Mod. Phys. 44 (1972) 320. 
[12] Mirea M., Poenaru D.N. et Greiner W., Three-dimensional dynamics of nuclear decay modes, Z. Phys. A 349 (1994) 39.

[13] Wapstra A.H. et Audi G., The 1983 atomic mass evaluation, Nucl. Phys. A 432 (1985) 1.

[14] Shicheng Wang, Snowden-Ifft D., Price P.B., Moady K.J, et Hulet E.K., Heavy-fragment radioactivity of ${ }^{238} \mathrm{Pu}$ : $\mathrm{Si}$ and $\mathrm{Mg}$ emission, Phys.Rev. C 39 (1989) 1647.

[15] Davies K.T.R. et Sierk A.J., Calculation of Coulomb energies for uniform charge distribution of arbitrary shape, J. Comput. Phys. 18 (1975) 311. 